\title{
Conteúdo energético das operárias da formiga cortadeira, Atta sexdens
}

\author{
Workers energy content in leaf-cutting ants, Atta sexdens
}

\section{Roberto da Silva Camargo ${ }^{\mathrm{I}^{*}}$ Juliane Floriano Santos Lopes $^{\mathrm{I}}$ Luiz Carlos Forti ${ }^{\mathrm{II}}$}

\section{RESUMO}

O conhecimento do conteúdo energético das operárias é essencial para mensurar a disponibilidade energética, responsável pela manutenção e execução de atividades essenciais para o crescimento da colonia de formigas. Mas pouco se conhece sobre o conteúdo energético das operárias e quanto estará disponível para realizar atividades de, por exemplo, escavar do ninho. O presente estudo determinou o teor de lipídeo e o conteúdo energético das operárias de formigas cortadeiras inativas, antes e pós atividade de escavação. Por meio da determinação do teor de lipídeos, pode-se calcular o conteúdo energético das operárias em repouso (paradas) e compará-las com as que escavaram. O teor de lipideo e conteúdo energético das operárias foram em média $9,1 \pm 0,8 \%$ e $111,31 \pm 54,71 \mathrm{~J}$, respectivamente, entretanto, as séries experimentais não diferiram significativamente. Adicionalmente, a taxa do fluxo catabólico, embasado na massa corporal das

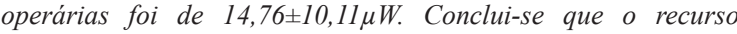
energético para a escavação do ninho não é proveniente de reserva de lipídeos corporal e, dessa forma, o conteúdo energético das operárias não alterou antes e pós-atividade de escavação.

Palavras-chave: teor de lipídeos, taxas metabólicas, comportamento, escavação do ninho.

\section{ABSTRACT}

The knowledge of the workers energy content is essential to measure the energy availability for maintenance and performance of activities essences for the colony growth. But little is known about the workers energy content, and how much will be available to carry out activities, for example, the excavation of the nest. The present study determined the lipid content and energy content of the worker ants inactive before and after excavation activity. Through the lipids determination, it can be calculated the energy content of workers resting (standing) with those which excavated. The lipid content and energy content of the workers were on average $9.1 \pm 0.8 \%$ and energy content of the workers was on average $111.31 \pm 54.71 \mathrm{~J}$, respectively, however, the experimental series did not differ significantly. Additionally, catabolic flux rate based in workers body mass was $14.76 \pm 10.11 \mu \mathrm{W}$. It was concluded that the energy resource for the excavation of the nest is not coming from reserve lipid body, and thus the energy content of the workers did not change before and after excavation activity.

Key words: lipid content, metabolic rates, behavior, digging the nest.

\section{INTRODUÇÃO}

O conteúdo energético já foi estudado em rainhas de formigas, em especial, aquelas pertencentes à tribo Attini (SEAL \& TSCHINKEL, 2007; SEAL, 2009; FUJIHARA et al., 2012). Em rainhas menores de Attini mais basais, verificou-se que Trachymyrmex septentrionalis apresenta $25 \%$ do seu peso seco constituído por gordura e Cyphomyrmex rimosus tem 11\% (SEAL \& TSCHINKEL, 2007). Já nas espécies mais derivadas (Atta sexdens e Atta laevigata), devido à fundação claustral, a rainha armazena uma grande reserva de gorduras, cerca de $40 \%$ de seu peso, que serão utilizados nessa fase da vida da colônia (DELLA LUCIA et al., 1994). Posteriormente, as rainhas recuperam seu peso e reservas lipídicas, com até $6,4 \%$ de gordura aos 12 meses (CAMARGO \& FORTI, 2013). Porém, não se sabe sobre a variação do teor de lipídeos e conteúdo energético das operárias de formigas cortadeiras, uma casta responsável por diversas atividades dentro da colônia, consequentemente, com uma alta atividade metabólica.

\footnotetext{
'MirmecoLab, Comportamento e Biologia Animal, Universidade Federal de Juiz de Fora (UFJF), Rua José Lourenço Kelmer, s/n, 36036-900, Juiz de Fora, MG, Brasil. E-mail: camargobotucatu@yahoo.com.br. *Autor para correspondência.

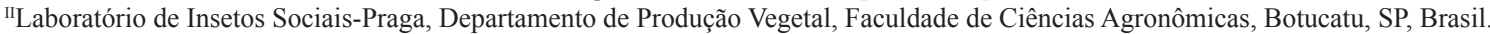
Recebido 22.02.13 Aprovado 27.05.13 Devolvido pelo autor 08.08.13 CR-2013-0238.R1
} 
As operárias são responsáveis por todas as tarefas necessárias para o desenvolvimento da colônia, portanto, cada atividade compreenderá um custo energético. As operárias têm inúmeras tarefas, que compreendem desde o cultivo do jardim de fungo, assistência à prole, escavação do ninho, defesa e forrageamento (ANDRADE et al., 2002; CAMARGO et al., 2007; CAMARGO et al., 2012). Entretanto, não se conhece o quão energeticamente é custoso para as operárias tais atividades rotineiras da colônia.

A proposta do estudo foi determinar o conteúdo energético das operárias da formiga cortadeira Atta sexdens rubropilosa, em repouso e em diferentes períodos de atividade escavatória. $\mathrm{O}$ presente artigo serve como base para estudos adicionais de mensuração das atividades executadas pelas formigas, bem como das respectivas taxas metabólicas para elas.

\section{MATERIAL E MÉTODOS}

Escavação do ninho pelas operárias

Cinco colônias de laboratório da formiga cortadeira Atta sexdens rubropilosa foram utilizadas para a retirada de operárias médias (largura da cabeça: 1,2 a 1,6mm), responsáveis pela escavação do ninho (CAMARGO et al., 2012). As séries experimentais foram:

a) Parada 0 hora ( $\mathrm{P} 0)$ : as operárias eram coletadas dos ninhos, sacrificadas e congeladas imediatamente para a determinação de lipídeos totais $(\mathrm{N}=10)$.

b) Parada 24 horas (P24): as operárias eram coletadas dos ninhos, permanecendo isoladas por 24 horas em recipiente, sendo posteriormente mortas por congelamento para a determinação de lipídeos totais $(\mathrm{N}=10)$.

c) Parada 48 horas (P48): as operárias eram coletadas dos ninhos, permanecendo isoladas por 48 horas em recipiente, sendo posteriormente mortas por congelamento para a determinação de lipídeos totais $(\mathrm{N}=10)$.

d) Parada 72 horas (P72): As operárias eram coletadas dos ninhos, permanecendo isoladas por 72 horas em recipiente, sendo posteriormente congeladas para a determinação de lipídeos totais $(\mathrm{N}=10)$.

e) Escavação 24 horas (E24): as operárias eram coletadas dos ninhos, dispostas em um ambiente com solo para escavar por 24 horas, sendo posteriormente mortas por congelamento para a determinação de lipídeos totais $(\mathrm{N}=10)$.

f) Escavação 48 horas (E48): as operárias eram coletadas dos ninhos, dispostas em um ambiente com solo para escavar por 48 horas, sendo posteriormente mortas por congelamento para a determinação de lipídeos totais $(\mathrm{N}=10)$.

g) Escavação 72 horas (E72): as operárias eram coletadas dos ninhos, dispostas em um ambiente com solo para escavar por 72 horas, sendo posteriormente mortas por congelamento para a determinação de lipídeos totais $(\mathrm{N}=10)$.

Os recipientes experimentais de escavação ( $10 \mathrm{~cm}$ de profundidade por $20 \mathrm{~cm}$ de diâmetro) foram preenchidos com solo do campus da Universidade (Latossolo), coletado a $60 \mathrm{~cm}$ de profundidade (densidade do solo $=1,6 \mathrm{~g} \mathrm{~cm}^{-3}$; conteúdo de água: 5,4\%) (STEIN \& XAVIER, 1984).

Determinação de lipídeos

Foi utilizada uma adaptação ao método de extração de COOK et al. (2010), em que os insetos foram imersos em solvente orgânico até atingirem um peso constante. Seguindo essa metodologia, as operárias foram imersas em $10 \mathrm{~mL}$ de pentano, durante 24 horas, e individualmente fechadas. Após esse período, o solvente foi removido e as operárias permaneceram durante 24 horas a $70^{\circ} \mathrm{C}$ em uma estufa. Posteriormente, foi determinada sua massa em balança analítica. O procedimento foi repetido por 172 horas de extração. Os valores foram expressos em porcentagem $(\%)$ em relação à massa seca das operárias.

O conteúdo energético das formigas foi obtido pela multiplicação da massa magra por $18,87 \mathrm{~J}$ $\mathrm{mg}^{-1}$ e pela massa gorda por $39,33 \mathrm{~J} \mathrm{mg}^{-1}$ (PEAKIN, 1972). Usando a taxa metabólica da equação alométrica $\mathrm{MR}=973 \mathrm{M}^{0,856}$, em que $\mathrm{M}$ é a massa das formigas em gramas (LIGHTON et al., 2001), foi determinada a taxa de fluxo catabólico ou taxa metabólica padrão, para insetos inativos, expressa em $\mu \mathrm{W}$.

A massa fresca, conteúdo de lipídeos e o conteúdo energético foram submetidos à Análise de Variância (ANOVA) $(\alpha=0,05)$, utilizando o programa SigmaPlot 11.0.

\section{RESULTADOS}

A massa média das operárias de Atta sexdens rubropilosa foi de 7,401 $\pm 3,634 \mathrm{mg}$, não diferindo significativamente entre as séries experimentais $\left(\mathrm{F}_{6 ; 343}=1,519, \mathrm{P}=0,171\right)$. O conteúdo de lipídeos (mg) também não diferiu entre as séries experimentais $\left(\mathrm{F}_{6 ; 343}=0,840, \mathrm{P}=0,540\right)$ (Tabela 1).

$\mathrm{O}$ conteúdo energético das operárias foi em média $111,31 \pm 54,71 \mathrm{~J}$, também não diferindo entre as séries experimentais $\left(\mathrm{F}_{6 ; 343}=1,334, \mathrm{P}=0,241\right)$ 
Tabela 1 - Média da massa ( $\mathrm{mg} \pm$ desvio padrão), da porcentagem de lipídeos e do conteúdo energético (J) das operárias de Atta sexdens rubropilosa submetidas a séries experimentais sem escavação $(\mathrm{P})$ e com escavação $(\mathrm{E})$ por diferentes períodos de tempo $(0,24$, $48,72 \mathrm{~h}$ )

\begin{tabular}{|c|c|c|c|c|c|c|c|}
\hline & $\mathrm{P} 0$ & $\mathrm{P} 24$ & P48 & P72 & E24 & $\mathrm{E} 48$ & E72 \\
\hline Massa fresca (vivo) (mg) & $7,5 \pm 4,8$ & $7,6 \pm 3,5$ & $8,6 \pm 4,5$ & $6,5 \pm 3,4$ & $7,1 \pm 2,9$ & $7,4 \pm 2,7$ & $7,0 \pm 3,5$ \\
\hline Massa seca (mg) & $2,8 \pm 1,8$ & $3,0 \pm 1,38$ & $3,5 \pm 1,83$ & $2,8 \pm 1,46$ & $2,8 \pm 1,14$ & $3,1 \pm 1,13$ & $2,8 \pm 1,4$ \\
\hline Massa magra (mg) & $2,6 \pm 1,67$ & $2,7 \pm 1,24$ & $3,2 \pm 1,67$ & $2,5 \pm 1,30$ & $2,5 \pm 1,01$ & $2,8 \pm 1,02$ & $2,6 \pm 1,3$ \\
\hline Conteúdo de lipídeo (mg) & $0,2 \pm 0,13$ & $0,3 \pm 0,14$ & $0,3 \pm 16$ & $0,3 \pm 0,16$ & $0,2 \pm 0,13$ & $0,3 \pm 0,11$ & $0,2 \pm 0,1$ \\
\hline Porcentagem de lipídeo (\%) & $8,0 \pm 6,5$ & $10,4 \pm 8,3$ & $9,2 \pm 6,4$ & $10,0 \pm 7,8$ & $9,2 \pm 6,9$ & $8,7 \pm 7,8$ & $8,5 \pm 5,6$ \\
\hline Conteúdo Energético $(\mathrm{J})$ & $107,1 \pm 69,4$ & $112,1 \pm 55,4$ & $129,4 \pm 65,8$ & $104,5 \pm 58,2$ & $104,5 \pm 38,3$ & $115,6 \pm 44,2$ & $105,9 \pm 51,2$ \\
\hline
\end{tabular}

(Tabela 1). A taxa do fluxo catabólico embasado na massa corporal das operárias que permanecem paradas foi de $14,76 \pm 10,11 \mu \mathrm{W}$, ou seja, 0,0148 J.

\section{DISCUSSÃO}

Os resultados obtidos demonstram que o conteúdo energético das operárias não foi afetado diretamente com a atividade de escavação (Tabela 1). Resultados similares já haviam sido observados em rainhas de Atta sexdens, em que a atividade de escavação não afetou o seu conteúdo energético (FUJIHARA et al., 2012). Esse resultado permite concluir que o recurso energético para a atividade de escavação não é originário das reservas de lipídeos, e sim de outra fonte, como carboidratos.

Diversas atividades utilizam hidratos de carbono, tais como o voo, sendo considerado uma das atividades de maior gasto energético, cerca de consumindo cerca de 20-100 vezes mais energia que a taxa metabólica padrão (ROCES \& LIGHTON, 1995). JUTSUM \& QUINLAN (1978) verificaram que $21 \%$ da massa seca dos alados de formigas cortadeiras são carboidratos e estes são totalmente consumidos após o vôo nupcial. Em Formica lugubris, demonstrou-se que o carboidrato (estocado como glicogênio) é a principal fonte de energia para o voo nupcial (PASSERA et al., 1989).

Adicionalmente, o conteúdo energético de uma rainha de formiga cortadeira, no dia da revoada, é de 6110.01J (FUJIHARA et al., 2012), cerca de 55 vezes o conteúdo energético das operárias médias, ou seja, as escavadoras do ninho. A maior parte do conteúdo energético provém do corpo gorduroso, localizado no abdômen das formigas. Seu armazenamento ocorre no interior de trofócitos e oenócitos (PEREIRA ROLLO \& CAMARGO MATHIAS, 2006; ROMA et al., 2006), os quais se prendem firmemente aos órgãos, através de ramos traqueais.

Coluna 1, Linha 42: trocar porém, o conteúdo energético gasto durante a atividade de escavação não foi detectado por embora, o gasto energético da atividade de escavação não tenha sido detectado (STAURENGO DA CUNHA \& CRUZ LANDIM, 1983). Cabe ressaltar que esse é o primeiro relato sobre o conteúdo energético das operárias escavadoras de formigas cortadeiras, porém, o conteúdo energético gasto durante a atividade de escavação não foi detectado.

O fluxo catabólico ou taxa metabólica padrão das operárias em repouso foi de $14,76 \pm 10,11 \mu \mathrm{W}$, ou seja, 0,0148J. Comparativamente, em formigas nectarívoras (Camponotus rufipes), a taxa metabólica padrão é de $24,62 \mu \mathrm{W}$, sendo o custo de transporte de néctar até o ninho 5 a 6 vezes a taxa metabólica padrão (SCHILMAN \& ROCES, 2005). Outro exemplo é Atta sexdens, cuja atividade de corte das folhas é energeticamente cara, com taxa metabólica até 30 vezes maior em relação ao repouso da operária (ROCES \& LIGHTON, 1995). Provavelmente, o ato de escavar o ninho inclui as duas atividades mencionadas acima, cortar a matriz do solo e, posteriormente, efetuar o tranporte para deposição do pellet de solo na superfície (CAMARGO et al., 2012).

Com base nos resultados, pode-se concluir que o recurso energético para a escavação do ninho não é obtido a partir da reserva de lipídeos, haja vista que o conteúdo energético das operárias não difere antes e pós-atividade de escavação. O presente artigo serve como base para estudos de mensuração das atividades executadas pelas formigas, bem como de suas respectivas taxas metabólicas.

\section{AGRADECIMENTOS}

O primeiro autor agradece ao Conselho Nacional de Desenvolvimento Científico e Tecnológico (CNPq) pela bolsa concedida pela modalidade Pós-doutorado Júnior. J.F.S. Lopes agradece à Fundação de Amparo à Pesquisa do Estado de Minas Gerais (FAPEMIG) (APQ 00342-12).

\section{REFERÊNCIAS}

ANDRADE, A.P.P. et al. Behavior of Atta sexdens rubropilosa (Hymenoptera: Formicidae) workers during the preparation of the leaf substrate for symbiont fungus culture. Sociobiology, v.40, 
n.2, p.293-306, 2002. Disponível em: <http://cat.inist.fr/?aModel $\mathrm{e}=$ afficheN\&cpsidt=13808817> . Acesso em: 06 dez. 2012. doi: 10.1111/j.1439-0418.2006.01129.x.

CAMARGO, R.S. et al. Nest digging by leaf-cutting ants: effect of group size and functional structures. Psyche, v.1 p.14, 2012. Disponível em: <http://www.hindawi.com/journals/ psyche/2012/426719/>. Acesso em: 05 mar. 2012. doi: $10.1155 / 2012 / 426719$

CAMARGO, R.S.; FORTI, L.C. Queen lipid content and nest growth in the leaf cutting ant (Atta sexdens rubropilosa). Journal of Natural History, v.47, n.1-2, p.65-73, 2013 Disponível em: $<$ http://www.tandfonline.com/doi/abs/10.10 80/00222933.2012.738836>. Acesso em: 21 fev. 2013 . doi: $10.1080 / 00222933.2012 .738836$

CAMARGO, R.S. et al. Age polyethism in the leaf-cutting ant Acromyrmex subterraneus brunneus Forel, 1911 (Hymenoptera: Formicidae). Journal of Applied Entomology, v.131, p.139-145, 2007. Disponível em: <http:/doi:10.1111 /j.1439-0418.2006.01129.x>. Acesso em: 06 dez. 2012. doi: 10.1111/j.1439-0418.2006.01129.x.

COOK, S.C.et al. Colony-level macronutrient regulation in ants: mechanisms, hoarding and associated costs. Animal Behaviour, v.79, p.429-437,2010. Disponível em: <http://doi:10.1016/j. anbehav.2009.11.022>. Acesso em: 06 dez. 2012. doi:10.1016/j. anbehav.2009.11.022

DELlA LUCIA, T.M.C. et al. Perda de peso de rainhas de Atta durante a fundação e o estabelecimento das colônias. Revista Brasileira Biologia, v.55, n.4, p.533-536, 1994.

FUJIHARA, R.T. et al. Lipids and energy contends in the bodies of queens of Atta sexdens rubropilosa Forel (Hymenoptera: Formicidae): pre and post nuptial flight. Revista Brasileira de Entomologia, v.56, n.1, p.73-75, 2012. Disponível em: <http:/ dx.doi.org/10.1590/S0085-56262012005000015>. Acesso em: 06 dez. 2012. doi: http://dx.doi.org/10.1590/S008556262012005000015 .

JUTSUM, A.R.; QUINLAN, R.J. Flight and substrate utilisation in laboratory-reared males of Atta sexdens. Journal Insect Physiology, v.24, p.821-825, 1978. Disponível em: <http://dx.doi. org/10.1016/0022-1910(78)90102-6>. Acesso em: 06 dez. 2012. doi: http://dx.doi.org/10.1016/0022-1910(78)90102-6.

LIGHTON, J. et al. Low metabolic rate in scorpions: implications for population biomass and cannibalism. Journal of Experimental Biology, v.204, p.607-613, 2001. Disponível em: <http://jeb. biologists.org/content/204/3/607.short>. Acesso em: 21 fev. 2013. doi: http://jeb.biologists.org/content/204/3/607.short.

PASSERA, L. et al. Carbohydrates as energy source during the flight of sexuals of the ant Formica lugubris (Hymenoptera: Formicidae). Entomologial Generalis, v.15, v.25-32, 1989.
PEAKIN, G.J. Aspects of productivity in Tetramorium caespitum L. EkolOgie Polka, v.20, p.55-63, 1972.

PEREIRA ROLLO, J.; CAMARGO MATHIAS, M.I. Morphohistochemical characterization of the perivisceral fat body in royal and worker female castes in different ages of Atta sexdens rubropilosa ants (Hymenoptera, Formicidae). Sociobiology, v.47, p.519-530, 2006. Disponível em: $<$ http://cat.inist.fr/?aModele $=$ aff icheN\&cpsidt=17659597>. Acesso em: $06 \mathrm{dez}$. 2012. doi: http:// dx.doi.org/10.1016/0022-1910(78)90102-8.

ROCES, F.; LIGHTON, J.R.B. Larger bites of leaf-cutting ants. Nature, v.373 p.373, 1995. Disponível em: <http://www.nature. com/nature/journal/v373/n6513/abs/373392a0.html>. Acesso em: 06 dez. 2012. doi:10.1038/373392a0.

ROMA, G.C.et al. Fat body in some genera of leaf-cutting ants (Hymenoptera: Formicidae). Proteins, lipids and polysaccharides detection. Micron, v.37, p.234-242, 2006. Disponível em: $<$ http:// www.sciencedirect.com/science/article/pii/S0968432805001824>. Acesso em: 06 dez. 2012. doi: http://dx.doi.org/10.1016/j. micron.2005.10.012.

SCHILMAN, P.E.; ROCES, F. Energetics of locomotion and load carriage in the nectar feeding ant, Camponotus rufipes. Physiological Entomology, v.30, p.332-337, 2005. Disponível em: <http://onlinelibrary.wiley.com/doi/10.1111/ j.1365-3032.2005.00464.x/>. Acesso em: 06 dez. 2012. doi: 10.1111/j.1365-3032.2005.00464.x.

SEAL, J.N. Scaling of body weight and fat content in fungus gardening ant queens: does this explain why leaf-cutting ants found claustrally? Insectes Sociaux, v.56, p.135-141, 2009. Disponível em: <http://doi:10.1007/s00040-009-0002-8>. Acesso em: $06 \mathrm{dez}$. 2012. doi: 10.1007/s00040-009-0002-8.

SEAL, J.N.; TSCHINKEL, W.R. Energetics of newly-mated queens and colony founding in the fungus-gardening ants Cyphomyrmex rimosus and Trachymyrmex septentrionalis (Hymenoptera: Formicidae). Physiological Entomology, v.32, p.8-15, 2007. Disponível em: $<$ http://onlinelibrary.wiley.com/do i/10.1111/j.1365-3032.2006.00534.x>. Acesso em: 06 dez. 2012. doi: 10.1111/j.1365-3032.2006.00534.x.

STAURENGO DA CUNHA, M.A.; CRUZ-LANDIM, C. Modificações histológicas e histoquímicas do corpo gorduroso de rainhas de Atta sexdens rubropilosa Forel (Hymenoptera, Formicidae) durante o primeiro ciclo reprodutivo. Acta Biológica Paranaense, v.12, p.11-22, 1983. Disponível em: $<$ http://ojs.c3sl. ufpr.br/ojs-2.2.4/index.php/acta/article/viewArticle/873>. Acesso em: 21 fev. 2013.

STEIN, C.; XAVIER, G.F. Does substrate density affect the nest depth of female Atta sexdens rubropilosa (Forel, 1908) (Hymenoptera: Formicidae). Brazilian Journal Medical Research, v.17, n.3-4, p. 297-300, 1984. 